\title{
Eosinophilic esophagitis and esophageal atresia: coincidence or causality?
}

\author{
Karen V. Stave Salgado, M.D. ${ }^{a}$ and Ana M. Rocca, M.D. ${ }^{a}$
}

\begin{abstract}
Eosinophilic esophagitis is an immune-mediated chronic disease of the esophagus characterized by symptoms related to esophageal dysfunction and tissue eosinophilia. In the endoscopy, the esophageal mucosa may appear normal or show exudates, rings, edema, furrows, and strictures. Its management is based on elimination diet, topical corticosteroids and/or esophageal dilation. Atresia is the most common congenital alteration of the esophagus; it requires surgical repair and poses potential complications, such as gastroesophageal reflux, strictures, and esophageal dysmotility. Up to 2015, 48 cases of eosinophilic esophagitis and esophageal atresia were reported, with dysmotility, reflux, and long-term acid suppression involvement. Prevalent clinical signs include dysphagia, difficulty eating, and reflux symptoms, so an esophagogastroduodenoscopy with biopsy is recommended to rule out associated eosinophilic esophagitis in patients with esophageal atresia and persistent symptoms before performing an esophageal dilation and / or anti-reflux surgery. If eosinophilic esophagitis is confirmed, it should be managed with the corresponding drugs, and the subsequent therapeuticapproach will depend on its clinical course.

Key words: eosinophilic esophagitis, esophageal atresia, dysphagia.
\end{abstract}

http: / / dx.doi.org/10.5546/ aap.2018.eng.e61

To cite: Stave Salgado KV, Rocca AM. Eosinophilic esophagitis and esophageal atresia: coincidence or causality? Arch Argent Pediatr 2018;116(1):e61-e69.

a. Department of Gastroenterology, Hospital de Pediatría SAMIC "Prof. Dr. Juan P. Garrahan," Buenos Aires, Argentina.

E-mail address:

Karen V. Stave Salgado, M.D.: kstave@gmail. com

Funding:

None.

Conflict of interest: None.

Received: 3-1-2017 Accepted: 7-11-2017

\section{GLOSSARY}

EA: esophageal atresia.

EGD: esophagogastroduodenoscopy.

EoE: eosinophilic esophagitis.

ESPGHAN: European Society for

Paediatric Gastroenterology,

Hepatology and Nutrition.

GERD: gastroesophageal reflux

disease.

hpf: high-power field.

IL: interleukin.

NASPGHAN: North American Society

for Pediatric Gastroenterology,

Hepatology and Nutrition.

PPI-REE: proton pump inhibitor responsive esophageal eosinophilia.
PPIs: proton pump inhibitors. TEF: tracheoesophageal fistula. UGI: upper gastrointestinal series.

\section{INTRODUCTION}

Eosinophilic esophagitis (EoE) is a chronic, immune-mediated disease of the esophagus, characterized by signs and symptoms related to esophageal dysfunction and eosinophil-predominant tissue inflammation. ${ }^{1,2}$

Esophageal atresia (EA) with or without tracheoesophageal fistula (TEF) is a congenital anomaly of the esophagus. The treatment of choice is surgical repair with potential late complications such as dysphagia, gastroesophageal reflux, strictures, and dysmotility. ${ }^{3-5}$

An increased risk of EoE has been described in patients with successful repair of EA secondary to gastroesophageal reflux disease (GERD), esophageal dysmotility, and the genetic similarities common to both conditions: the mutations in the Forkhead box (FOX) gene cause congenital anomalies, such as EA. In addition, FOXF1 genes are present in the promoter region of inflammation, including eotaxin-3, which is increased in EoE; therefore, Gorter et al. proposed that patients with EA may have a greater risk for developing EoE. Based on the above, EoE should be considered when making a differential diagnosis in children with repaired EA who have recurrent strictures, persistent dysphagia, and refractory symptoms related to gastroesophageal reflux., ${ }^{3,6}$

\section{EOSINOPHILIC ESOPHAGITIS}

EoE is a chronic, immunemediated clinical-pathological entity of the esophagus. Diagnostic criteria include: $2,7,8$ 
- symptoms related to esophageal dysfunction;

- 15 or more eosinophils per high-power field (hpf) in the endoscopic biopsy of the esophageal mucosa; and

- exclusion of other causes of esophageal eosinophilia, including proton pump inhibitorresponsive esophageal eosinophilia (PPI-REE).

EoE was first described in 1990 and its prevalence ranges from 1 to 5 per 10000 individuals in the United States and Europe. EoE may affect all age groups but it is more common among men (3:1) and Caucasians, and it occurs in $8-10 \%$ of children with suspected anti-reflux-refractory GERD. ${ }^{3,9}$

EoE is believed to be a Th2 cell-mediated entity triggered by food and / or environmental antigens. There is an increase in interleukin (IL)-5 and IL-13, which are responsible for eosinophil differentiation and maturation, and for stimulating the esophageal epithelium to produce eotaxin-3, that recruits eosinophils. Such activated eosinophils release multiple factors that promote local inflammation and tissue injury.

There may be at least 3 genes with potential functional alterations in EoE; these genes encode eotaxin-3, thymic stromal lymphopoietin, and calpain-14. The esophageal tissue of patients with EoE shows dilated interepithelial spaces, altered epithelial barrier function, downregulation of proteins associated with barrier function (filaggrin and zonulin-1) and adhesion molecules (desmoglein-1) (Figure 1). Such altered epithelial permeability can lead to a permissive environment that enhances antigen presentation, which in turn leads to eosinophil recruitment. ${ }^{9}$

The clinical manifestations vary depending on age: infants may have difficulty eating (food refusal, regurgitation, and vomiting) and growth failure. In preschoolers and school children, nausea, vomiting, heartburn, and chest pain are predominant. Adolescents have abdominal pain, dysphagia, food impaction, and gastroesophageal reflux. ${ }^{1,10}$

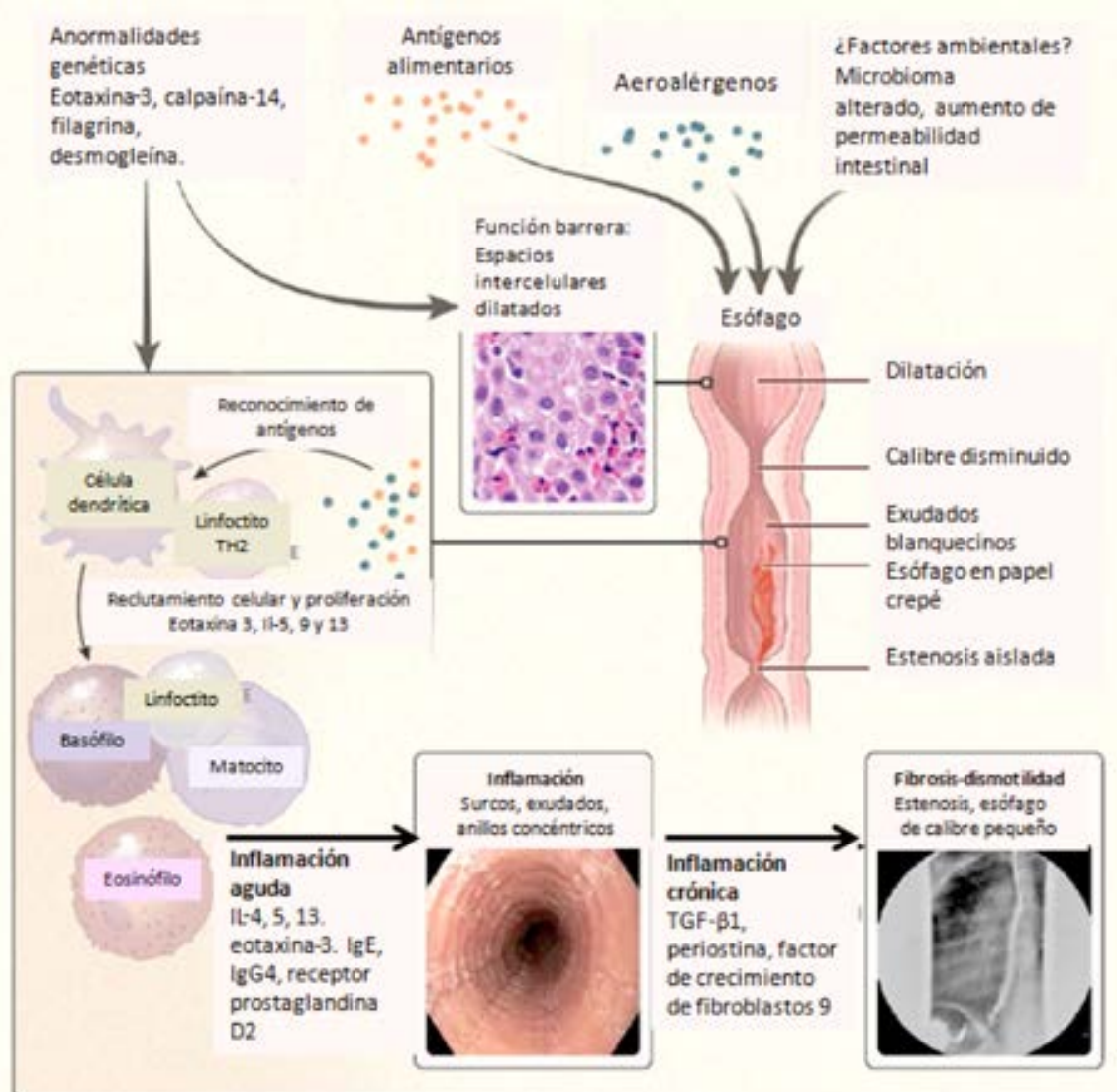

Eosinophilic esophagitis is an immune-mediated disease in which eosinophils are recruited to the esophagus. The consequences of this inflammation include stricture formation with proximal dilation. Adapted from N Engl J Med 2015. 373: 1640-1648. 
Children with EoE more commonly have atopy: $30-50 \%$ have asthma; $50-75 \%$, allergic rhinitis; and more than $50 \%$, a family history of allergy. ${ }^{7}$

The esophagogastroduodenoscopy (EGD) usually shows edema, fixed rings (corrugated esophagus or trachealization), exudates, longitudinal furrows, strictures or small-caliber esophagus, friable mucosa, and easy bleeding, also called crêpe-paper mucosa. Approximately 7-32\% of patients may have a normal endoscopy. In pediatrics, the most common findings are edema and exudates ${ }^{8,10,11}$ (Figure 2).

The recommendation is to collect at least 2-4 biopsies of the upper and lower third of the esophagus because it usually shows a patchy involvement, and of the gastric antrum and duodenum to rule out eosinophilic gastroenteritis. ${ }^{1,12}$ The histological marker of EoE is the presence of 15 or more eosinophils per hpf, with a $100 \%$ sensitivity and a $96 \%$ specificity. Eosinophil degranulation, eosinophil microabscesses, basal cell hyperplasia, and lamina propria fibrosis may also occur 7,10 (Figure 3). Other causes of esophageal eosinophilia include GERD, PPI-REE, fungal or bacterial infection, Crohn's disease, celiac disease, vasculitis, hypereosinophilic syndrome, and connective tissue disorders. ${ }^{7,8,12}$

The treatment goals are complete resolution of clinical symptoms, eosinophilic inflammation, and esophageal remodeling. To this end, dietary and pharmacologic interventions are used: ${ }^{1,7,10}$

\section{Diet therapy}

Diet therapy is considered an effective first line treatment for all children with EoE. There are three dietary approaches:

a) Elemental diet: including amino acid-based formulas. It is used for 4 weeks as an alternative for patients with multiple food allergies, growth failure or severe refractory disease.

b) Six-food elimination: it empirically eliminates the six most common allergenic foods (milk, soy, wheat, eggs, nuts / peanuts, and fish / shellfish) for 8-12 weeks.

c) Targeted diet: elimination of foods based on allergy test results for 8-12 weeks.

\section{Non-dietary aspects}

These include pharmacologic treatment and endoscopic dilation.

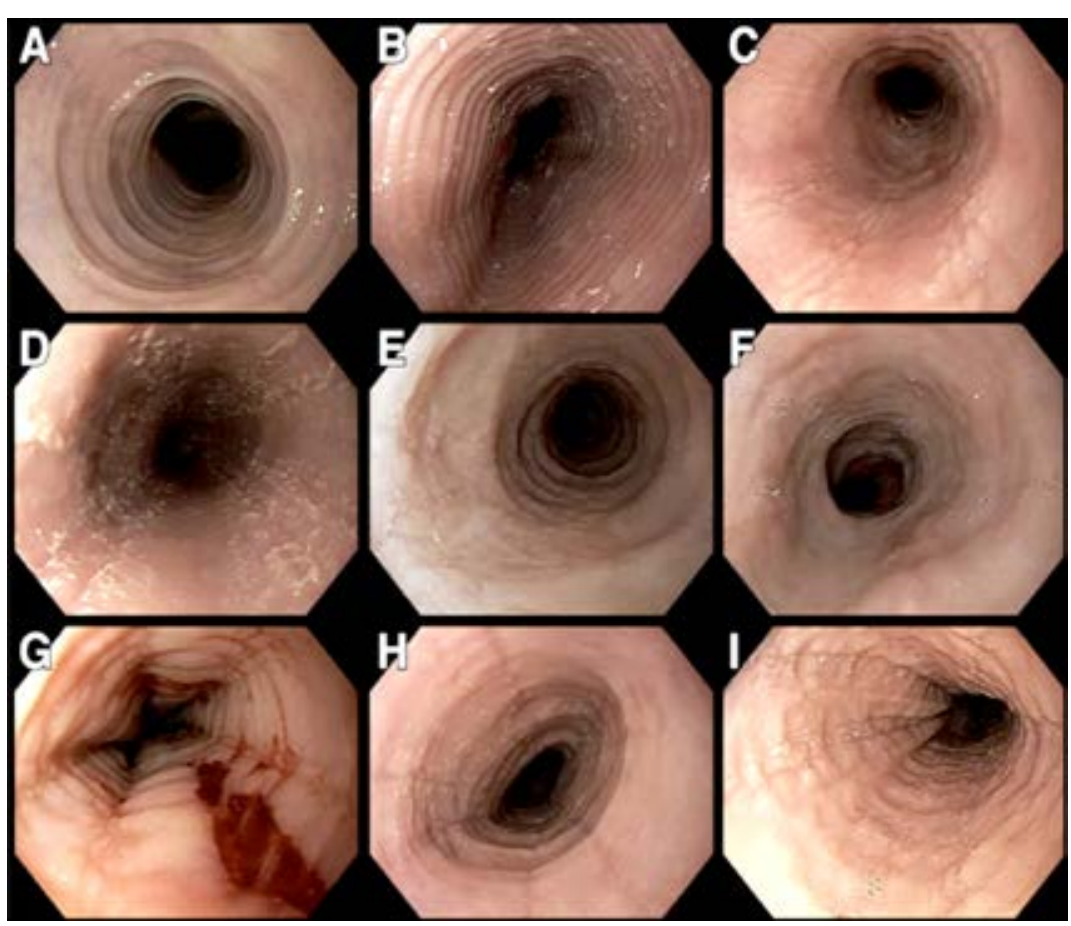

A. Fixed rings (trachealization). B. Transient rings (felinization). C. Linear furrows. D. Exudates. E. Esophageal narrowing with mucosal edema. F. Focal stricture. G. Crêpe-paper mucosa. H. Rings, furrows, exudates. I. Rings, furrows, edema. Adapted from Gastroenterology 2014;1-17. 
a) PPIs: once esophageal eosinophilia is determined, PPIs should be started at $1 \mathrm{mg} /$ $\mathrm{kg} /$ dose (up to $40 \mathrm{mg}$ ), twice daily, for 8-12 weeks, and a second EGD should be done after treatment. If eosinophilia is resolved, it may be GERD or PPI-REE; if eosinophilia persists, EoE diagnosis is confirmed (Figure 4). b) Topical corticosteroids: viscous budesonide and swallowed fluticasone are used for 4-12 weeks, alone or in combination with diet. Budesonide dosage: $1 \mathrm{mg} /$ day in patients younger than 10 years and $2 \mathrm{mg} /$ day in patients older than 10 years (up to $2.8 \mathrm{mg}$ and $4 \mathrm{mg}$, respectively). Fluticasone: $88-440 \mu \mathrm{g}$ 2-4

FIGURE 3. Histologic characteristics of eosinophilic esophagitis

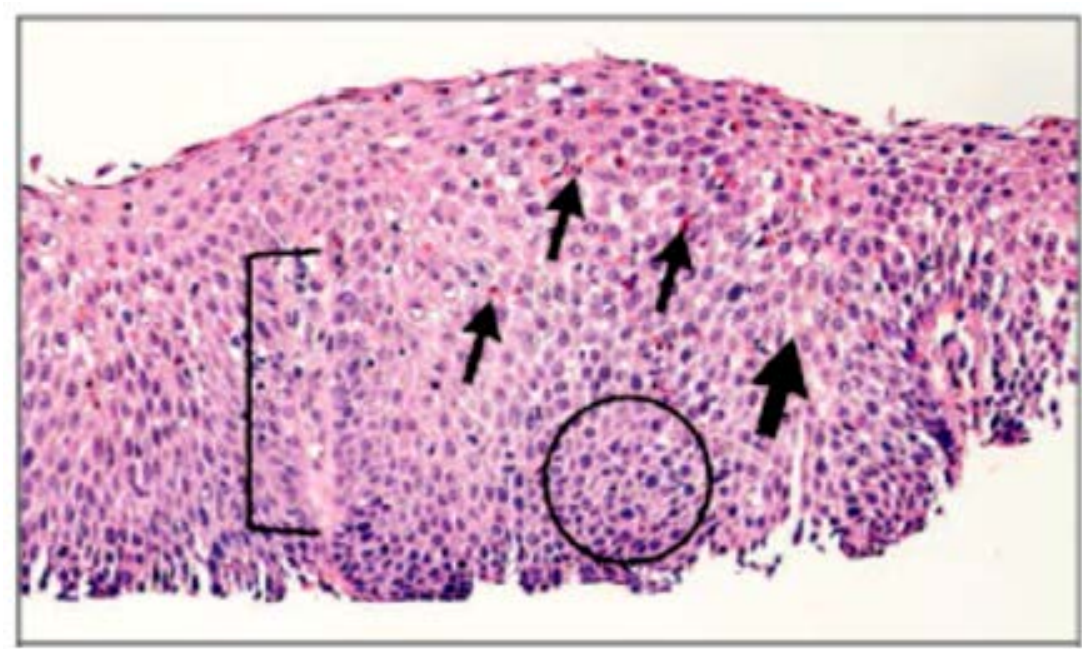

Papillary elongation (bracket), numerous eosinophils (thin arrows), dilated intercellular spaces (thick arrow), basal zone hyperplasia (circle). Adapted from N Engl J Med 2015;373:1640-1648.

FIGURE 4. Algorithm for the evaluation of children and adolescents with symptoms suggestive of eosinophilic esophagitis

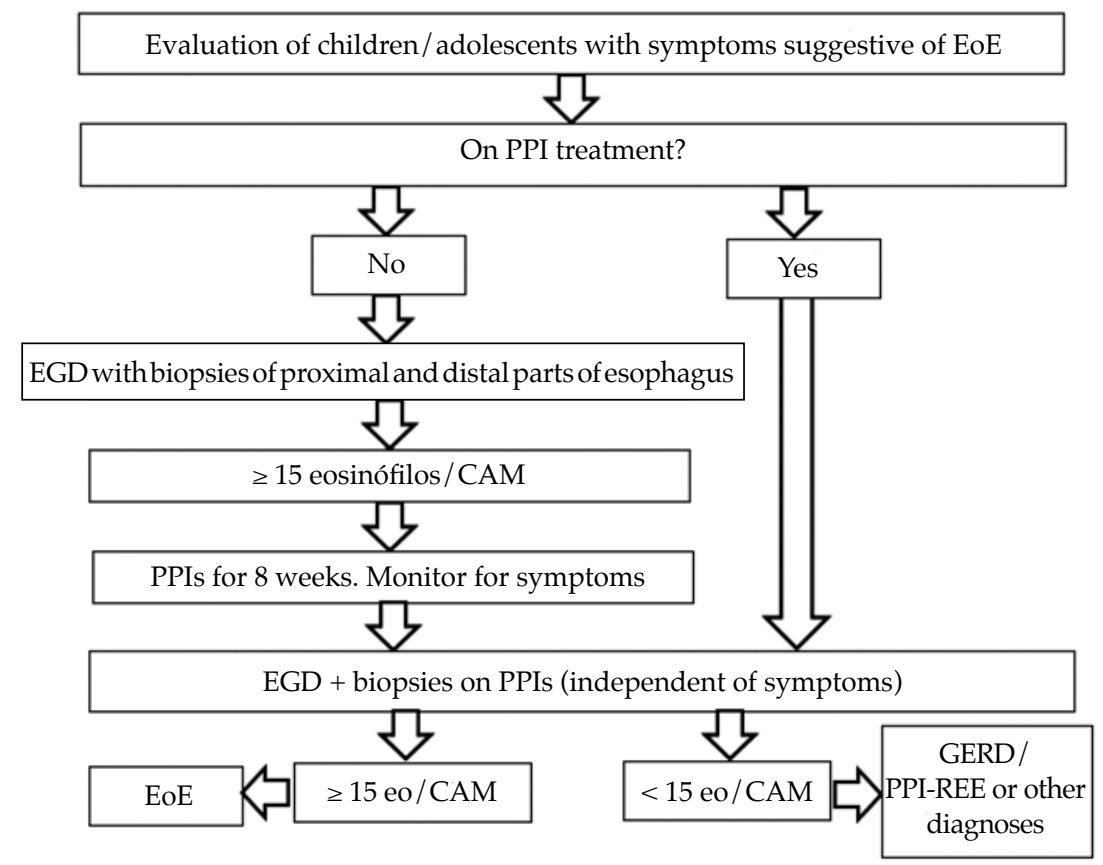

EoE: eosinophilic esophagitis. eo: eosinophils. hpf: high-power field. PPIs: proton pump inhibitors.

EGD: esophagogastroduodenoscopy. GERD: gastroesophageal reflux disease.

PPI-REE: proton pump inhibitor-responsive esophageal eosinophilia. Adapted from J PediatrGastroenterolNutr 2014;58:107-118. 
times / day in children and 440-880 $\mu \mathrm{g}$ twice/ day in adolescents. Patients should not drink, eat or rinse their mouth for 30 minutes.

c) Systemic corticosteroids: indicated for severe dysphagia, food impaction, dehydration, weight loss, and esophageal stricture. Oral prednisone, $1-2 \mathrm{mg} / \mathrm{kg} /$ day (up to $60 \mathrm{mg} /$ day); symptoms improve after 7-10 days of treatment.

d) Other treatments: sodium cromoglycate, leucotriene receptor antagonists, thiopurines, and biologics have not demonstrated their usefulness.

e) Dilation: recommended for persistent esophageal stricture in spite of other therapies used.

Figure 5 describes an algorithm for the management of children and adolescents with EoE.

\section{ESOPHAGEAL ATRESIA}

EA is the most common congenital alteration of the esophagus, with an incidence of 1 in 25004500 live births. It is more predominant among males and Caucasians, and in case of the following risk factors: primigravida women, advanced maternal age, and twin pregnancy. ${ }^{13,14}$ Also, 50\% of cases have associated congenital malformations; gastrointestinal malformations occur in 25\%; and the VACTER sequence (vertebral, anorectal, cardiac, tracheo-esophageal, and renal defects) has been observed in $10-20 \% .^{14,15}$
More than 100 types of EA have been reported; the most frequent type is EA with lower TEF. Vogt's original classification (1929), modified by Ladd (1944) and Gross (1953), has been used to date; it includes six types -Type I: EA without fistula. Type II: EA with upper TEF. Type III: EA with lower TEF. Type IV: EA with double fistula. Type V: isolated TEF (H-type). Type VI: congenital esophageal stricture. ${ }^{14}$

EA is commonly diagnosed in the first 24 hours of life, although it can be suspected antenatally due to the presence of polihydramnios, a prominent esophageal pouch or a small or absent stomach during the third trimester of gestation. At birth, EA should be considered in case of excessive salivation, regurgitation, cough, respiratory distress, cyanosis or inability to pass a catheter into the stomach. H-type fistula, which is commonly diagnosed at a later time, presents with cough while feeding, recurrent pneumonia, cyanosis, and intermittent abdominal distension. ${ }^{15,16}$ Diagnosis is confirmed with a chest and neck X-ray (anterior and lateral view), by passing a radiopaque catheter or a catheter with water-soluble contrast media to guide its position. Chest and abdominal $X$-rays show the presence of intestinal air in the case of type III atresia or its absence in the case of type $\mathrm{I}^{14}$

The surgery consists in closing the TEF and performing an end to end anastomosis of esophageal ends using a thoracoscopy or thoracotomy technique. "Long gap esophageal

FIGURE 5. Algorithm for the management of children and adolescents with eosinophilic esophagitis

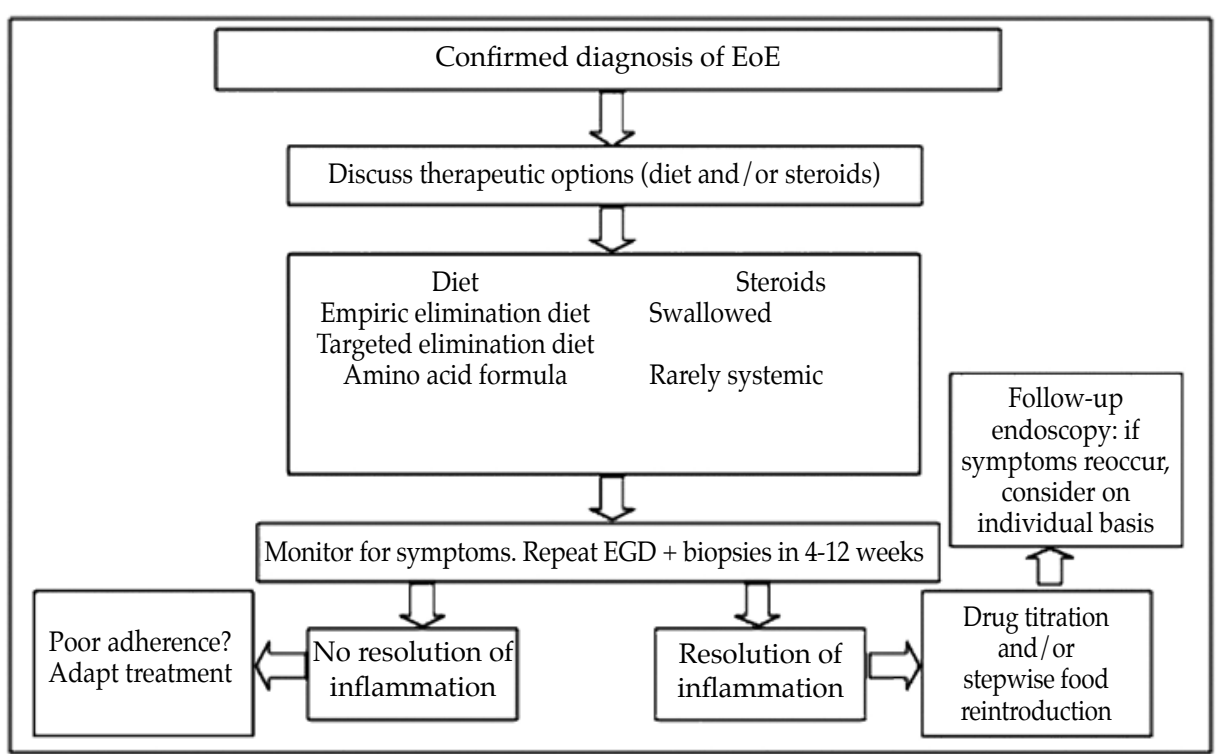

EoE: eosinophilic esophagitis. EGD: esophagogastroduodenoscopy. Adapted from J Pediatr Gastroenterol Nutr 2014;58:107-118. 
atresia" refers to atresia that precludes the creation of an end to end anastomosis; it is resolved with a sequential extrathoracic esophageal elongation (Kimura's technique) or replacement with stomach, colon or small intestine. Improvements in surgical techniques together with advances in neonatal care have enhanced patient prognosis and, at present, survival is more dependent on the presence or absence of other malformations than on EA-TEF itself. $^{13,15}$

Early post-operative complications may occur, including wound dehiscence, esophageal stricture and refistula, and late complications, which may affect the gastrointestinal (gastroesophageal reflux, stricture, dysmotility) and respiratory systems. GERD is one of the most common gastrointestinal complications, occurring in up to $58 \%$ of patients. It is secondary to esophageal dysmotility, short esophagus, lower esophageal sphincter pressure, and chronic lung disease. ${ }^{4,17,18}$ Dysmotility is the result of intrinsic factors related to abnormal esophagus development or partial denervation during surgery. Motor patterns were described in a cohort of children with EA using a high resolution manometry. The study included 40 patients with a median age of 8 years (11 months- 18 years) and found motility alterations in all of them: isolated distal contractility $(47 \%)$, aperistalsis $(38 \%)$, and pressurization $(15 \%)$. However, these patterns failed to predict the presence or severity of dysphagia. ${ }^{19,20}$

Dysphagia, which was observed in $21-84 \%$ of patients, is evidenced in food refusal, food impaction, difficulty swallowing, odinophagia, cough, choking, pneumonia, vomiting, and malnutrition. It may be attributed to inflammatory and anatomic causes such as peptic esophagitis or stricture, eosinophilic esophagitis, congenital or anastomotic stricture, post-fundoplication obstruction, vascular anomalies, diverticulum in anastomosis, and mucosal bridges. In the absence of these causes, esophageal dysmotility is still the most widely accepted explanation. ${ }^{19}$

Recently, the European Society for Paediatric Gastroenterology, Hepatology and Nutrition (ESPGHAN) and the North American Society for Pediatric Gastroenterology, Hepatology and Nutrition (NASPGHN) published the Guidelines for the Evaluation and Treatment of Gastrointestinal and Nutritional Complications in EA-TEF, and recommended that any patient with EA and GERD/gastroesophageal reflux should receive acid suppression with PPIs since the neonatal period up to 1 year old or older, depending on ongoing symptoms and peptic complications. They recommended that dysphagia should be assessed with contrast studies and EGD with biopsies. Among asymptomatic patients, routine EGD should be done before discontinuing PPI treatment, before 10 years old, and at the time of transition to an adult health care center. Although severe dysmotility predisposes to post-fundoplication complications, some patients with EA may benefit from this surgical procedure if they have recurrent anastomotic stricture, PPI-refractory GERD, and long-term dependence of transpyloric feeding. Pre-operative assessment should include 24-hour reflux monitoring, upper gastrointestinal series (UGI) and gastrointestinal endoscopy. A pH-metry is required to confirm and quantify acid reflux; a UGI helps to diagnose hiatal hernia and associated congenital stricture, assess the anatomic features of the cardiac region, and exclude other intestinal malformations; an EGD is necessary to conduct a gross examination, collect biopsy specimens, screen for peptic esophagitis, EoE, and Barrett's esophagus. ${ }^{19}$

\section{ESOPHAGEAL ATRESIA AND EOSINOPHILIC ESOPHAGITIS}

No controlled studies on the prevalence of EoE in patients with EA have been carried out. Up to 2015,48 cases had been reported. The largest series was published by Dhaliwal et al., which established a $17 \%$ incidence (18 cases). ${ }^{18}$

Several hypothesis explain the highest frequency of EoE and EA: 1) alterations in esophageal motility lead to a prolonged exposure to potential allergens, thus increasing the risk of local sensitization; 2) GERD causes mucosal injury and may impair the mucosal barrier function and predispose to EoE; 3) food impaction secondary to dysmotility and esophageal stricture associated with EA may damage the mucosa and cause eosinophilic infiltration; 4) long-term exposure to acid suppression, which is common among children with EA, may increase food protein allergenicity; 5) the 16q24 microdeletion involving the FOX transcription factor gene, in humans, has been associated with EA, cardiac, anorectal, renal, gastrointestinal, and vertebral anomalies. Curiously, FOXF1 genes have been identified in mice, and FOXF1 binding sites are present not only in the promoter region of genes that are critical for mesenchymal proliferation but also 
in the inflammation promoter region, including those for eotaxin and interleukin. The expression of eotaxin-3 is increased in patients with EoE. Gorter et al. have proposed that mutations in the FOX gene not only cause congenital malformations in the esophagus and lung but may also predispose to EoE, so patients with EA may be at a high risk for EoE. 3,6,21

The above mentioned observations suggest that EoE should be taken into consideration when making a differential diagnosis in patients with EA who have dysphagia and refractory gastroesophageal reflux symptoms before considering anti-reflux surgery. ${ }^{3,21}$

The age of onset of EoE and EA ranges from 18 months to 12.5 years, and this entity is more common among men, except for the study by Dhaliwal et al., where it was predominant among women (1.6:1). In relation to the clinical presentation, this study found a significantly higher incidence of GERD symptoms, such as vomiting and dysphagia, among patients with
EoE compared to those without EoE. $6,18,22$ As it is known in EoE, in all series, associated atopy was frequent (Dhaliwal: 50\%; Batres: 66\%; Gorter: $50 \%)^{3,18,23}$ A normal endoscopy, linear furrows, edema, and white exudates were described as endoscopic findings. ${ }^{18,21,22}$

Multiple biopsies of both the proximal and distal parts of the anastomosis are recommended for EoE diagnosis. ${ }^{19}$

In EA, anastomotic stricture occurs in 5-15\% of patients, mainly in the first year of life. The etiology of this entity is multifactorial: early strictures are caused by tension and relative ischemia at the anastomosis site, whereas late strictures are secondary to acid reflux. Anastomotic strictures are short and discrete, unlike allergic strictures, which may be short or long. In EA, strictures are dilated routinely; however, in EoE, it has been demonstrated that topical corticosteroids may reverse the fibrotic subepithelial process. Esophageal strictures have been reported in patients with EA and EoE at a

FIGURE 6. Algorithm for the investigation and treatment of persistent dysphagia in patients with esophageal atresia

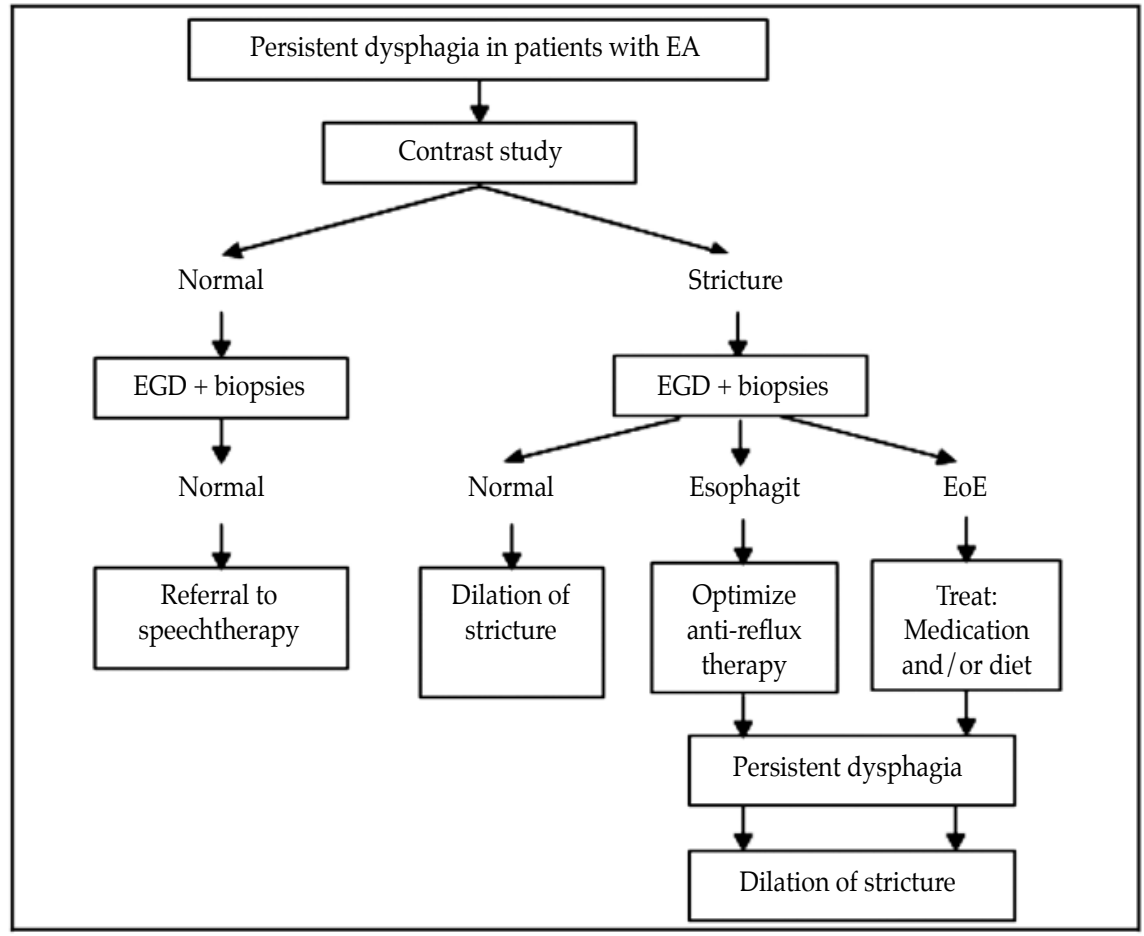

Persistent dysphagia in patients with EA should be assessed with esophagogram and EGD + multiple biopsies.

Based on the findings, endoscopic dilation, anti-reflux medication, or elimination diet with or without topical corticosteroids are proposed.

EA: esophageal atresia. EGD: esophagogastroduodenoscopy. EoE: eosinophilic esophagitis. Adapted from Eur J PediatrSurg 2015;25:336-344 and Dis Esophagus 2014;27:340-347. 
$20 \%$ rate by Kassabian et al., a 50\% rate in the study conducted by Oliveira et al., a $100 \%$ rate by Batres et al., a 38\% rate by Dhaliwal et al., and a $83 \%$ rate by Yamada et al. $18,21,24$

In the study carried out by Dhaliwal et al., patients with EoE significantly required a fundoplication more frequently than those without EoE, whereas no differences were observed in terms of gastrostomy requirements. ${ }^{18}$

A retrospective study by Chan et al. assessed the outcomes of treatment for EoE in children with EA. The study included 20 patients with a mean age of 26 months at the time of diagnosis; 8 were treated with viscous budesonide (0.5-1 $\mathrm{mg}$ twice / daily); 5, with swallowed fluticasone (50-500 $\mu \mathrm{g}$ twice/daily); 1 , with an elimination diet; and 6, with combined therapy; all were receiving PPIs at the time of diagnosis. Esophageal dilation was reserved for persistent stricture in spite of EoE treatment. Results demonstrated that treatment improved dysphagia and reflux symptoms, and reduced eosinophil count, strictures, and the number of dilations required. ${ }^{25}$

For the management of EoE and EA, the ESPGHAN and the NASPGHAN recommend to consider the consensus established for EoE treatment in the general population..$^{1,19}$

In sum, several authors consider it is important to study dysphagia, recurrent strictures, difficulty eating, and PPI-refractory reflux symptoms in patients with EA, not only with a contrast study but also with an EGD and multiple biopsies before performing an esophageal dilation and anti-reflux surgery. If EoE is confirmed, it should be managed with the corresponding drugs and / or an elimination diet. Endoscopic dilation is reserved for patients with refractory strictures in spite of an adequate EoE and/or GERD treatment or if clinically indicated (Figure 6). ${ }^{6,18,19,25}$

\section{REFERENCES}

1. Papadopoulou A, Koletzko S, Heuschkel R, et al. Management Guidelines of Eosinophilic Esophagitis in Childhood. J Pediatr Gastroenterol Nutr 2014;58(1):107-18.

2. Liacouras CA, Furuta GT, Hirano I, et al. Eosinophilic esophagitis: updated consensus recommendations for children and adults. J Allergy Clin Immunol 2011;128(1):3-20.

3. Gorter RR, Heij HA, Van der Voorn JP, et al. Eosinophilic esophagitis after esophageal atresia: is there an association? Case presentation and literature review. J Pediatr Surg 2012;47(6):e9-13.

4. Pedersen RN, Markow S, Kruse-Andersen S, et al. Esophageal Atresia: Gastroesophageal functional follow-up in 5-15 years old children. J Pediatr Surg 2013;48(12):2487-95.

5. Gottrand M, Michaud L, Sfeir R, et al. Motility, digestive and nutritional problems in Esophageal Atresia. Paediatr
Respir Rev 2016;19:28-33.

6. Krishnan U. Eosinophilic Esophagitis in Children with Esophageal Atresia. Eur J Pediatr Surg2015;25(4):336-44.

7. Dellon E, Liacouras C. Advances in clinical management of eosinophilic esophagitis. Gastroenterology 2014;147(6):1238-54.

8. Dellon ES, Gonsalves N, Hirano I, et al. ACG clinical guideline: evidenced based approach to the diagnosis and management of esophageal eosinophilia and eosinophilic esophagitis. Am J Gastroenterol 2013;108(5):679-92.

9. Furuta GT, Katzka D. Eosinophilic Esophagitis. N Engl J Med 2015;373(17):1640-8.

10. Pierre R, Guisande A, Sifontes L, et al. Diagnóstico y tratamiento de la esofagitis eosinofílica en niños. Revisión de la literatura y recomendaciones basadas en la evidencia. Grupo de trabajo de la Sociedad Latinoamericana de Gastroenterología, Hepatología y Nutrición pediátrica (SLAGHNP). Acta Gastroenterol Latinoam2015;45(3):263-71.

11. Kia L, Hirano I. Advances in the endoscopic evaluation of eosinophilic esophagitis. Curr Opin Gastroenterol 2016;32(4):325-31.

12. Muir AB, Merves J, Liacouras CA. Role of Endoscopy in Diagnosis and Management of Pediatric Eosinophilic Esophagitis. Gastrointest Endoscopy Clin N Am 2016; 26(1):187-200.

13. Smith N.Oesophageal atresia and tracheo-oesophageal fistula. Early Hum Dev2014;90(12):947-50.

14. García H, Gutiérrez MF. Manejo multidisciplinario de los pacientes con atresia de esófago. Bol Med Hosp Infant Mex2011;68(6):467-75.

15. Holland A, Fitzgerald D. Oesophageal atresia and tracheooesophageal fistula: current management strategies and complications. Paediatr Respir Rev 2012;11(2):100-7.

16. Kinottenbelt G, Skinner A, Seefelder C. Tracheo-oesophageal fistula (TOF) and oesophageal atresia (OA). Best Pract Res Clin Anaesthesiol 2010;24(3):387-401.

17. Shah R, Varjavandi V, Krishnan U. Predictive factors for complications in children with esophageal atresia and tracheoesophageal fistula. Dis Esophagus 2015;28(3):216-23.

18. Dhaliwal J, Tobias V, Sugo E, et al. Eosinophilic esophagitis in children with esophageal atresia. Dis Esophagus 2014;27(4):340-7.

19. Krishna U, Mousa H, Dall'Oglio L, et al. ESPGHANNASPGHAN Guidelines for the Evaluation and Treatment of Gastrointestinal and Nutritional Complications in Children with Esophageal Atresia-Tracheoesophageal Fistula. J Pediatr Gastroenterol Nutr 2016;63(5):550-70.

20. Lemoine C, Aspirot A, Le Henaff G, et al. Characterization of esophageal motility following esophageal atresia repair using high-resolution esophageal manometry. J Pediatr Gastroenterol Nutr 2013;56(6):609-14.

21. Kassabian S, Baez-socorr V, Sferra T, et al. Eosinophilic esophagitis in patients with esophageal atresia and chronic dysphagia. World J Gastroenterol 2014;20(47):18038-43.

22. Yamada Y, Nishi A, Kato M, et al. Esophagitis with Eosinophil Infiltration Associated with Congenital Esophageal Atresia and Stenosis. Int Arch Allergy Immunol 2013;161(Suppl 2):159-63.

23. Batres LA, Liacouras C, Schnaufer L, etal.Eosinophilicesophagitis associated with anastomotic strictures after esophageal atresia repair. J Pediatr Gastroenterol Nutr 2002;35(2):224-6.

24. Oliveira C, Zamakhshary M, Marcon P, et al. Eosinophilic esophagitis and intermediateesophagitis after tracheoesophageal fistula repair: a case series. J Pediatr Surg 2008;43(5):8104.

25. Chan LJ, Tan L, Dhaliwal J, et al. Treatment outcomes for eosinophilic esophagitis in children with esophageal atresia. Dis Esophagus 2016;29(6):563-71. 